\title{
Formation vector based shortest path-planning in CGF formation
}

\author{
Fei Jie, Zhaohan Lu, Baodi Xie \\ School of Computer Science and Technology \\ Beijing Institute of Technology \\ Beijing, China \\ jiefei2008@hotmail.com
}

\begin{abstract}
In order to improve the poor reality and bad flexibility of the mapping relationship which matched entities with aim locations in traditional approximation method, the formation vector shortest path-planning method was presented in this paper. By analyzing the lack of aim path-planning in the approximation method, shortest path-planning was discussed and was improved by introducing the formation vector and the idea of pheromone. Furthermore, the improved algorithm was applied in a CGF simulation system. The experimental results showed that the mapping relationship had better reality and rationality and the possibility of collision was significantly reduced than the traditional formation change process.
\end{abstract}

Keywords-computer generated forces; formation change; the approximation method; aim path-planning

\section{INTRODUCTION}

In military simulation, CGF team usually advanced in a particular formation [1] which was selected and changed according to the constraint condition and situation change in the battlefield. With the constant expansion of the simulation scale and the increase of the simulation entities, CGF formation change process had more practical value in CGF team simulation. In virtual battlefield, the formations of tank platoon included rank, column, wedge and so on, and every formation had different advantages and application ranges. Thus, the formation should be selected on the basis of battlefield situation. In a CGF team simulation system, whether the CGF team could select suitable formations and implement the formation changes had become an important sigh to measure the intelligence and reality of simulation systems.

There are two main methods to implement the formation change in a CGF team: direct changing method and the approximation method. Both the two methods were on the basis of the formations' mathematic descriptions. In traditional formation change problem, the standard formations were given by the tactical handbook, and the formations' mathematic descriptions were built and saved in the formation database. When the formation began to change, the CGF entities queried the database and got the formation descriptions, then moved to specified location or followed other entities according to the qualification in formation descriptions.
In this paper, the aim path planning was introduced to improve the formation changing process in the approximation method. Compared with traditional approximation method, this changing process could select a suitable location for the CGF entities in initial formation from the aim locations in new formation according to some specific conditions. This method could largely prevent the problems caused by fixed aim location selection in traditional method, and improve the flexibility and reality of formation change.

\section{Formation Change Process}

To complete the formation change, every CGF entity was required to update its attributes such as position, velocity and orientation according to the specific constraints of formation change, finally arrived at the appropriate location in final formation. There are two main methods to implement this process [2]:

(1) Direct changing method: Every CGF entity's aim location and orientation was computed before the formation change began. In the formation change process, all CGF entities moved to their aim locations with given velocities and orientations until the formation change completed.

(2) The approximation method: In the formation change process, a CGF entity was selected as benchmark entity and was drive along the formation vector. Other entities followed the benchmark entity, computed their locations in the new formation with the relative positions information, and constantly updated their velocities and orientations to approximate the aim locations.

In the two methods, the former did not match the actual situation in battlefield because it just considered the initial and final status of the formation change. The approximation method, in contrast, maintained control of all entities in the whole formation changing process, met the requirement of simulation, and was widely used in more CGF simulation system [3].

The mathematic description of CGF team formation is basis of formation change process. Taking tank platoon as an example, US Army tank platoon had 7 common battle formations which were shown in figure 1 . Every battle 

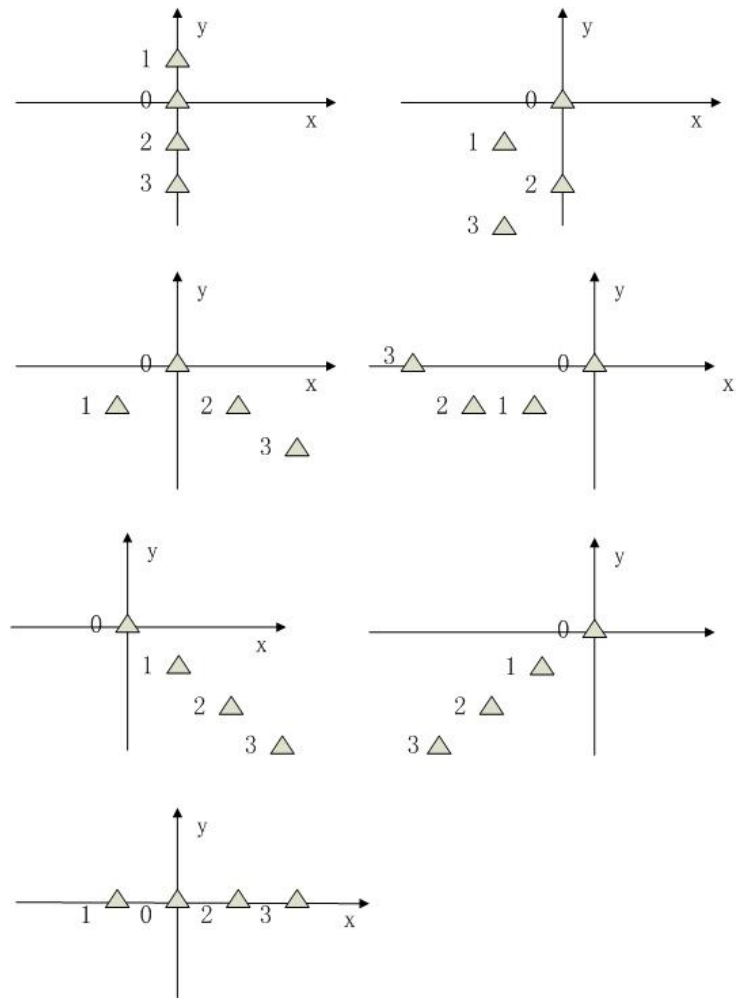

Figure 1. Seven common battle formations in US Army tactical handbook.

formation had its application range [4], such as the column formation was usually used to move quickly and across narrow fields; the stagger formation was usually used to move quickly and may encounter enemies. Taking tank 0 as the original point, taking formation vector as $\mathrm{y}$ axis positive direction, a local coordinate system was established. And the distance (d) from tank i to tank 0 (benchmark tank) and the angle $(\alpha)$ between the line which connected tank $i$ to tank 0 and $\mathrm{x}$ axis could be read in the tactical handbook. Then the coordinates of tank $\mathrm{i}$ was $\mathrm{P}_{\mathrm{i}}(\mathrm{d} \times \cos (\alpha), \mathrm{d} \times \sin (\alpha))$. If the position of tank 0 in global coordinate system were $P_{0}$

$(\mathrm{x}, \mathrm{y})$, the heading origination was $\beta$, and a follower tank moved from position $\mathrm{P}_{1}$ to position $\mathrm{P}_{1}$, then the position coordinates in new formation of this tank were:

$$
\begin{aligned}
P_{1}{ }^{\prime} \cdot x=P_{0} \cdot x+d \times \cos (\alpha) & \times \cos (\beta) \\
& +d \times \sin (\alpha) \times \sin (\beta) . \\
P_{1}{ }^{\prime} \cdot y=P_{0} \cdot y+d \times \cos (\alpha) & \times \sin (\beta) \\
+ & d \times \sin (\alpha) \times \cos (\beta)
\end{aligned}
$$

In the approximation method, the follower tank would set $\mathrm{P}_{1}{ }^{\prime}(\mathrm{x}, \mathrm{y})$ as its aim location, constantly updated its velocity and origination to approximate $\mathrm{P}_{1}{ }^{\prime}$, and arrived at $\mathrm{P}_{1}$ ' in the changing time $\mathrm{T}$ to complete the formation change.

As is apparent from the description above, the follower tank's aim location was determined by the benchmark tank's location information and the follower tank's relative position in new formation. That means, in new formation, every tank's aim location was specified by the tactical handbook. However, this kind of specifying was not always accordant with actual situation, and could even result in too long path and overmuch path crossing of follower tanks which would add complexity to collision avoidance.

To solve this problem, aim path planning was used to improve the formation changing process of the approximation method. Aim path planning could select a suitable location for each tank in initial formation from the aim locations in new formation according to some specific conditions (such as nearest distance, similar distance) so that it overcome the problems caused by inflexible location selection in a certain degree.

\section{FORMATION Vector BASED SHORTEST PATH- PLANNING}

In this section, aim path-planning method would be introduced to plan suitable locations for tanks from the aim locations in new formation.

\section{A. Classical Shortest Path-Planning}

In classical path planning, one of the common qualifications is to need the shortest path [5]. Thus, the shortest path-planning is one of common algorithms in aim path planning. Suppose there was a CGF team of $n$ tank entities which changed its formation from formation $\mathrm{A}$ to formation $\mathrm{B}$, then this process needed $\mathrm{n}$ selecting steps. In the ith selecting step, an entity was selected from $(n-i+1)$ entities in initial formation which needed to compute the distances from $(n-i+1)$ entities in initial formation to $(n-i+1)$ aim locations in new formation. The detailed process [6] was:

1) Compute the distance set $d_{i}$ between entity i's position and $\mathrm{n}$ locations in new formation.

2) Find the shortest distance $d_{i j}$ between entity i's position and $\mathrm{n}$ locations in new formation.

3) Find the minimum $d_{n}$ in $n$ entities' shortest distances.

From the steps above, it could be seen that classical shortest path-planning was trying to build a map which had the shortest distances between initial points and aim locations. So the crux of this algorithm was a large number of distance calculation and relevant sort work for them. However, the classical shortest path-planning still had too high time complexity for its application in the real time battlefield simulation. Besides, the distance between some last-matched entities and aim locations might be too long than others because the classical shortest path-planning was lack of the relative positions of positions in the formation. For these reasons, some improvements of the classical algorithm were necessary to adapt the application in military simulation.

\section{B. Formation Vector Based Shortest Path-Planning}

Formation vector was a unit vector that gave the driving direction of a formation. As it could be used to calculate the global coordinates of follower tanks in the approximation method, formation vector contained some structure 


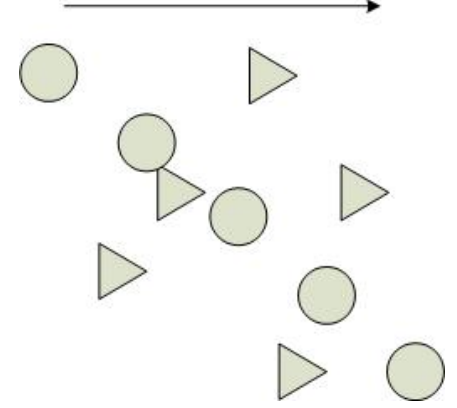

Figure 2. Relative positions of tanks in initial formation and final formation.

information of the formation and provided new minds for improving classical shortest path-planning. By watching the process of formation change, it was not hard to discover that the distribution orders of the entities along the direction of formation vector or the direction that perpendicular to formation vector in initial formation and new formation had changed little.

In order to make the mapping relation more reasonable and more real, the classical shortest path-planning needed some detail adjustments. As shown in figure 1, a local coordinate system was still established in final formation. Then the tanks in initial formation were translated in this coordinate system and their average coordinates was coincident with the original point. In figure 2 , the triangles represented the current positions of tanks and the circles represented the aim locations in final formation. After the relative positions were illustrated, formation vector was used to improve the entity-position matching process by doing shortest path-planning for the tanks in turn along the $\mathrm{x}$ axis or the $\mathrm{y}$ axis which depended on the features of the final formation.

Moreover, the pheromone theory of ant colony algorithm was referenced to reduce the time complexity in our algorithm. The pheromone, which was some chemical substances left by preceding ants, could be perceived and used to determine the moving direction by ants. In the foraging process, each ant would select the path which had denser pheromone and reinforced the pheromone on this path. In ant colony algorithm [7], the idea of pheromone was using prior experiences to guide the searching process to avoid detours. In our algorithm, every entity in initial formation and every position in new formation had a mark bit which show whether this entity (position) had been matched. If an entity had matched an aim location, the entity and position would be skipped in following calculation. That way, some unnecessary calculations were omitted and the calculation cost of the algorithm was reduced.

Based on the discussion above, the improved aim pathplanning could be described in the following steps:

1) Establish a local coordinate system in final formation and translate the entities' current positions to where the average coordinates was coincident with the original point.
2) In the initial formation, select an unmatched entity $\mathrm{i}$ along the $\mathrm{x}$ axis or the $\mathrm{y}$ axis, and compute the distance set $d_{i}$ between entity i's position and every unmatched location in new formation.

3) Find the minimum $d_{i j}$ in the distance set $d_{i}$ what you got in step 2. That means entity $i$ was matched with aim location $\mathrm{j}$.

4) Mark the entity $i$ and position $j$ as "matched", and jump to step 2 until all the entities had matched a aim location.

\section{IMPLEMENT AND APPLICATION}

In order to verify the rationality and validity of the algorithm, in this section some simulation experiments had been made in a CGF project to appraise the performance of algorithms. In the virtual battlefield, as was shown in figure 3 and 4 , a CGF team which consisted of 5 tanks was going to change the formation from stagger to wedge. In order to facilitate observation, some additional graphical representations were added in the scene. Those Arabic numerals at image right represented the aim locations in new formation. And the red lines that connected one tank and one position represented the mapping relationship in the formation change.

As was shown in figure 3, the traditional formation change process matched each tank with an aim location based only on entity ID so the matching lines had several intersections. More intersections means the tanks had a

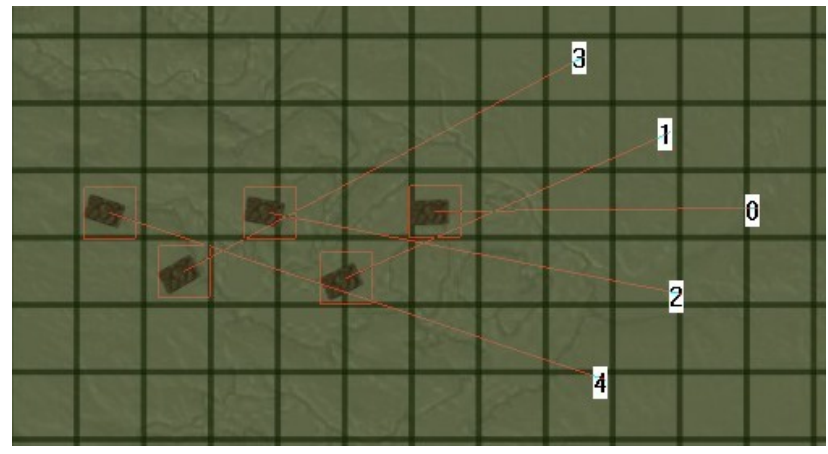

Figure 3. The entity-position matching result in classical formation change process

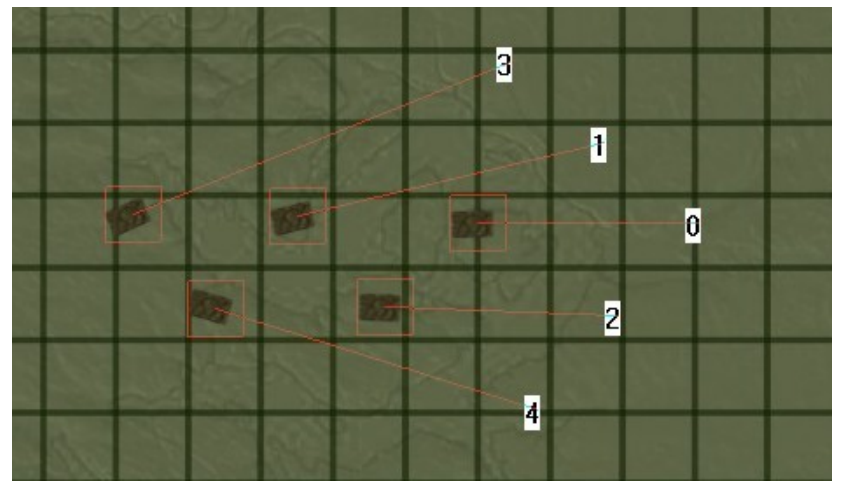

Figure 4. The entity-position matching result in formation vector based shortest path-planning. 
higher likelihood of colliding. In figure 4, formation vector based shortest path-planning was used to improve the formation change process. In this experiment, unmatched entities were selected along the direction of formation vector in step 2 of this algorithm. The experimental results showed that the number of intersections had significantly decreased and the mapping relationship had better reality and rationality.

\section{CONCLUSION}

This paper analyzed the formation change process and traditional approximation method in military simulation, discussed the possible problems because of lack of aim pathplanning, introduced the classical shortest path-planning and improved it, finally realized the formation vector based shortest path-planning method. Compared with the traditional approximation method, the formation vector based shortest path-planning had more flexibility to match aim locations in new formation with entities in initial formation. And the improved algorithm reduced much calculation cost than classical shortest path-planning.

In the application experiment, formation vector based shortest path-planning method was applied in a CGF simulation system. The experimental results showed that the mapping relationship had better reality and rationality and the possibility of collision was significantly reduced.
In order to improve further the approximation method, we will going to experiment on the algorithm by increasing the number of entities, and further reduce unnecessary calculation cost. Besides, for the formation change process in advance, the movement track which a follower entity approximates its aim location should be optimized to make it has better continuity.

\section{REFERENCES}

[1] P. Tabuada, G. J. Pappas, and P. Lima, "Motion Feasibility of MultiAgent Formations," IEEE Transactions On Robotics, vol.21, March. 2005, pp. 387-391

[2] L. G. Yang, G. Ji, Q. S. Guo and X. C. Wang, "The Simulation of Group Change in the Aggregated Computer Generated Forces," Computer Engineering and Applications, 23: 17-34, 2001

[3] T. Balch, R. C. Arkin, "Behavior-based formation control for multirobot teams", IEEE Transactions on Robotics \& Automation, vol. 12, Jun. 1998, pp. 926-939

[4] X. L. Kong, Q, Xue, and J. H. Song, "Research and Realization on Formation's Change of Tank CGF Unit" 4th Chinese Conference of Virtual Reality and Visualization, Aug. 2004, pp. 61-65

[5] R. W. Floyd, "Algorithm 97: Shortest Path", Communications of the ACM 18, 1968

[6] Y. Zhang, "Research on the virtual scheduling optimization methods of large scale theatrical performances" unpublished

[7] H. B. Duan, D. B. Wang, J. Q. Zhu, and X. H. Huang, "Development on Ant Colony Algorithm Theory and Its Application," Control and Decision, vol.19, Dec. 2004, pp. 1321-1326 